Supporting Information

\title{
Large Temperature-Independent Magnetoresistance without Gating Operation in Monolayer Graphene
}

Jihoon Jeon, ${ }^{\dagger}$ Duk Hyun Lee, ${ }^{\dagger}$ Yeon Soo Kim, ${ }^{\S}$ Hyun-Jong Chung, ${ }^{\dagger}$ Sung Ho Jhang, ${ }^{\dagger}$ Yongkyung Kwon, ${ }^{\dagger}$ Suyoun Lee ${ }^{\#, *}$ and Bae Ho Park ${ }^{\dagger, *}$

'Division of Quantum Phases \& Devices, Department of Physics, Konkuk University, 120

Neungdong-ro, Gwangjin-gu, Seoul 05029, Republic of Korea.

Korea Research Institute of Standards and Science, 267 Gajeong-ro, Yuseong-gu, Daejeon

34113, Republic of Korea.

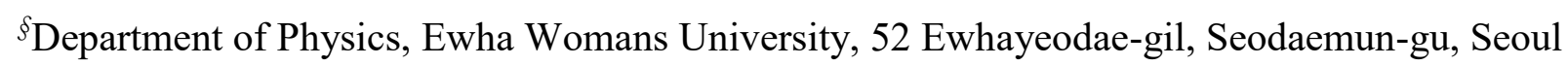
03760, Republic of Korea.

${ }^{\#}$ Center for Neuromorphic Engineering, Korea Institute of Science and Technology, 5 Hwarang-

ro 14-gil, Seongbuk-gu, Seoul 02792, Republic of Korea

*Corresponding authors; E-mail: slee_eels@kist.re.kr; baehpark@konkuk.ac.kr. 


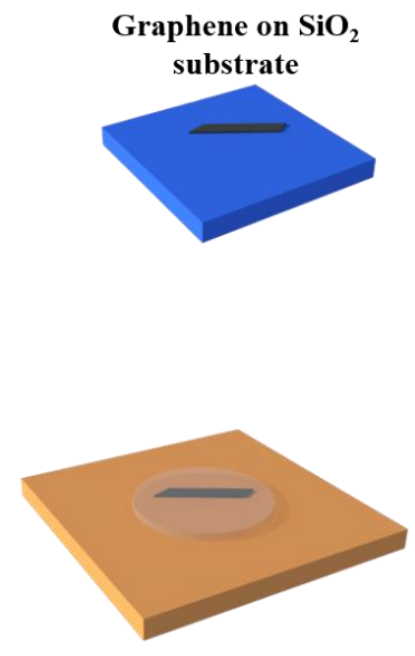

Lift-off UV tape

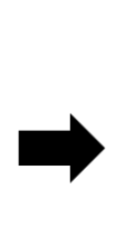

PMMA spin-coating
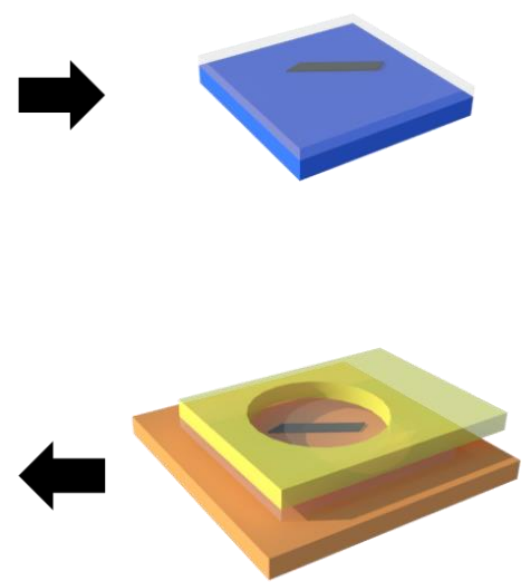

Transfer
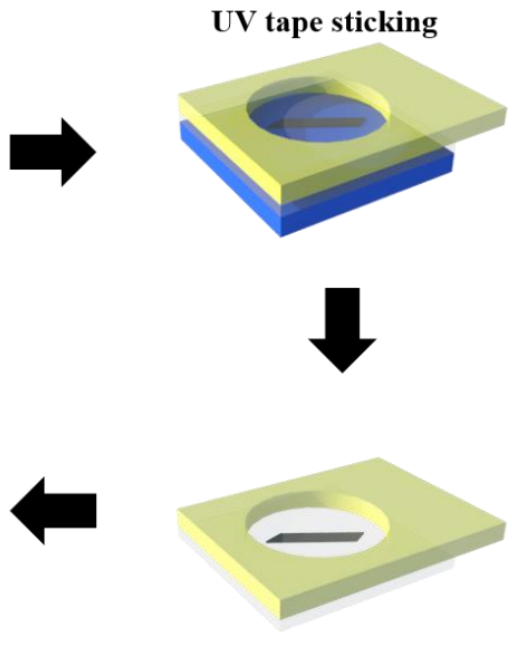

$\mathrm{SiO}_{2}$ etching

Figure S1. Simplified flow chart of the PMMA-supported transfer method for graphene onto target substrates.

Poly(methyl methacrylate) (PMMA) was spin-coated on top of graphene and electrodes at 4500 $\mathrm{rpm}$ for $45 \mathrm{~s}$ and heated at $180{ }^{\circ} \mathrm{C}$ for 2 min. Subsequently, ultraviolet (UV) tape, acting as a handling layer, was pasted on the PMMA/graphene membrane. The $\mathrm{SiO}_{2}$ substrate was etched with $\mathrm{KOH}$ solution and the UV tape/PMMA/graphene membrane was floated on the solution. After rinsing the membrane with distilled water and drying it under air for several hours, the UV tape was clamped onto the arm of a micromanipulator mounted on an optical microscope. Using the microscope to locate the position of the graphene optically on the $\mathrm{BiFeO}_{3}$ (BFO) nano-island array (BFO-NIA), the graphene was accurately aligned with the target position. Finally, a PMMAcoated graphene/BFO nano-island array (G/BFO-NIA) device was obtained by mechanically lifting off the UV tape. 
a

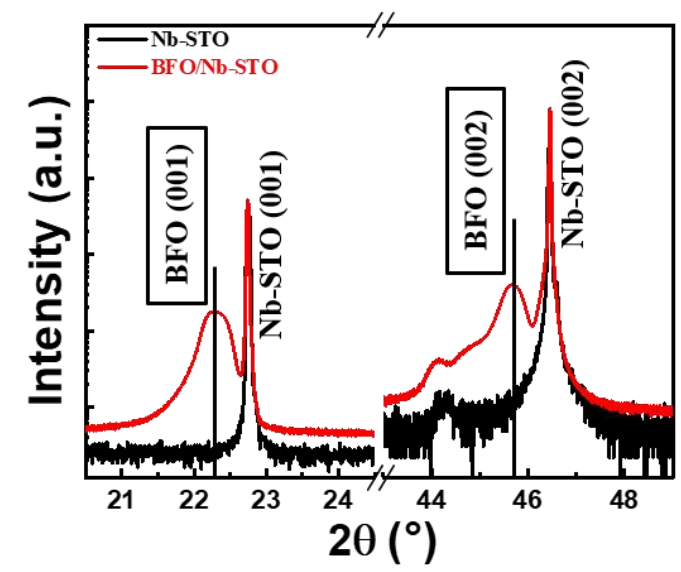

C

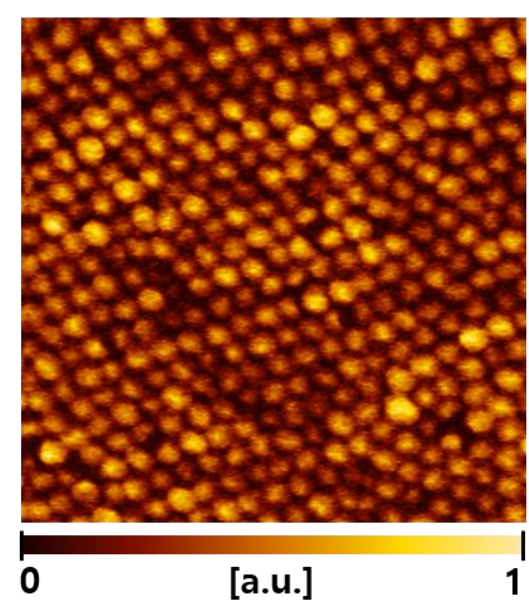

b

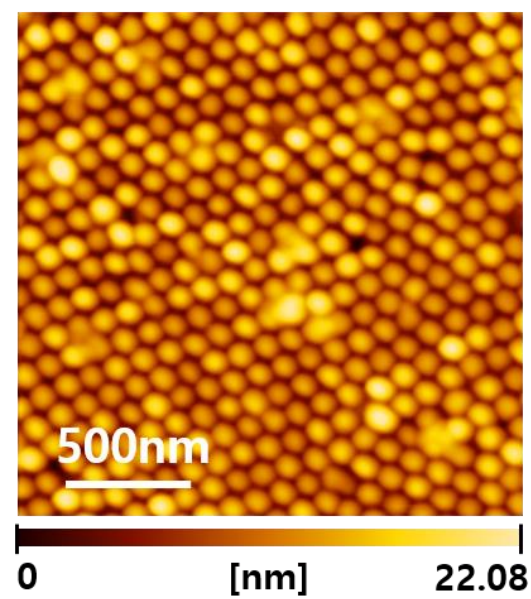

d

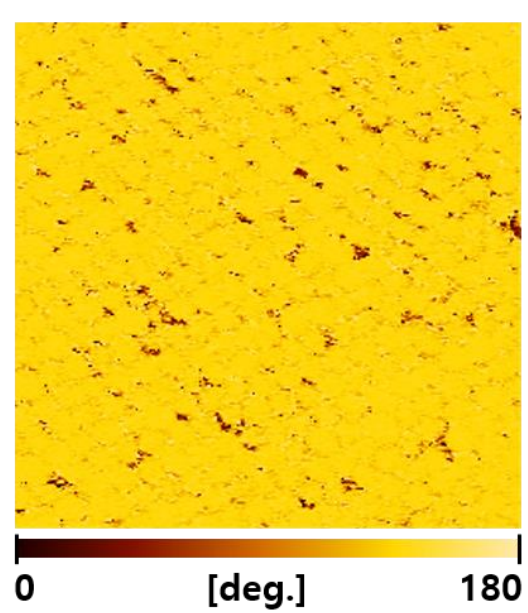

Figure S2. (a) HR-XRD patterns, (b) AFM topography image, (c) PFM amplitude image, and (d) PFM phase image obtained from the BFO nano-island array on Nb:STO (100) substrate.

We investigated the structural and local ferroelectric characteristics of the fabricated BFO-NIA using high-resolution X-ray diffraction (HR-XRD), atomic force microscope (AFM), and a piezoresponse force microscope (PFM), as shown in Figure S2. The BFO nano-islands were arranged in a regular manner, and the diameter of an individual BFO nano-island is $\sim 80 \mathrm{~nm}$. It is confirmed that the BFO nano-islands exhibit a single-crystalline rhombohedral structure, whose (001) direction is perpendicular to the Nb:STO substrate. From the BFO (002) peak position of the 
HR-XRD data, the out-of-plane lattice constant of the BFO is estimated to be $\sim 0.400 \mathrm{~nm}$. The single bright contrast in the PFM phase image implies that the as-grown BFO nano-islands consist of a downwardly aligned polarization.

a

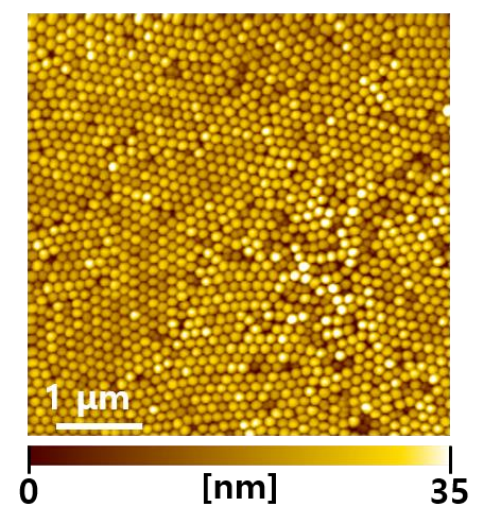

C

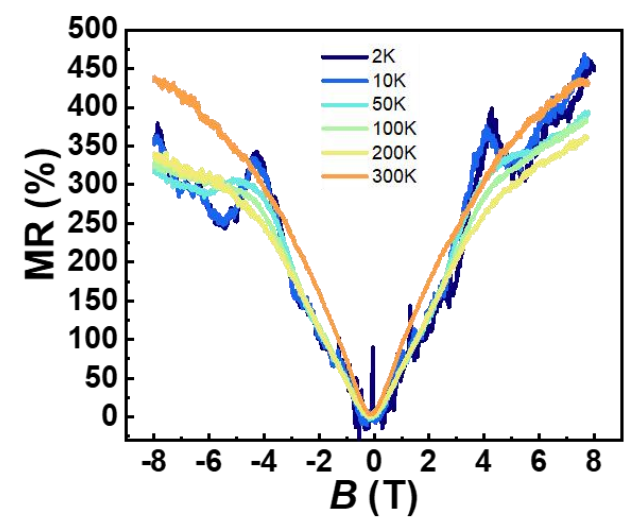

b

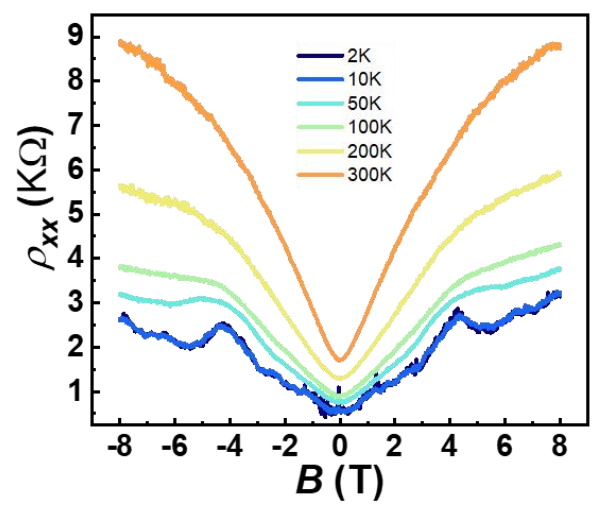

d

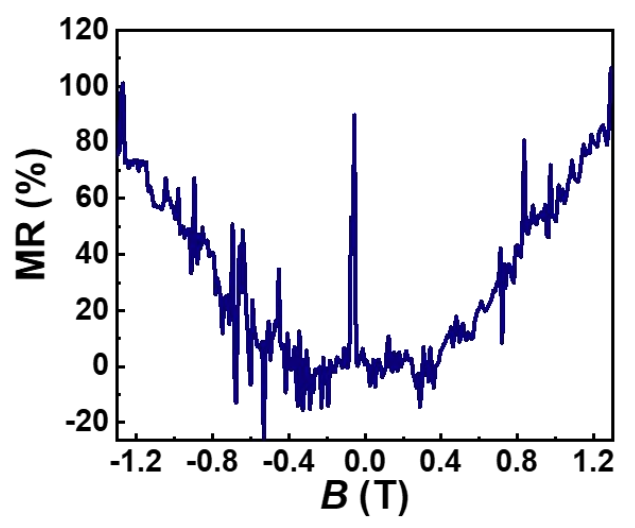

Figure S3. (a) AFM topography image of the STO-NIA. (b) $\rho_{x x}$ and (c) longitudinal MR of the G/STO-NIA structure at various temperatures $(2 \mathrm{~K}, 10 \mathrm{~K}, 50 \mathrm{~K}, 100 \mathrm{~K}, 200 \mathrm{~K}$, and $300 \mathrm{~K})$. (d) Weak localization like behavior at $2 \mathrm{~K}$. 
We fabricated a high-density array of $\mathrm{SrTiO}_{3}$ (STO) nano-islands on an STO/Nb:STO structure using an AAO template through PLD. The deposition temperature of STO-NIA is $600{ }^{\circ} \mathrm{C}$. All the fabrication conditions of a G/STO-NIA, except for the deposition temperature, are the same as a G/BFO-NIA. We obtained an AFM topography image (Figure S3a) of the STO-NIA. It can be seen that the diameter and arrangement of the STO nano-island are the same as the BFO nanoisland. Figure S3b shows $\rho_{x x}$ of the G/STO-NIA, which measured in the magnetic field range from $-8 \mathrm{~T}$ to $8 \mathrm{~T}$. Figure S3c presents the longitudinal MR properties of the G/STO-NIA. The MR curves show obvious quantum oscillations at low temperatures and the MR value is $\sim 440 \%$ when magnetic field of $8 \mathrm{~T}$ is applied at room temperature. The MR value decreases as the temperature increases from $2 \mathrm{~K}$ to $200 \mathrm{~K}$. On the other hand, as the temperature increases from $200 \mathrm{~K}$ to 300 $\mathrm{K}$, the MR value increases and shape of MR changes more linearly. The MR value of the G/STONIA is larger than that of G/BFO-NIA ( $210 \%$ at $8 \mathrm{~T})$ at room temperature. However, it exhibits a temperature dependent MR characteristic such as quantum phenomena at low temperatures and change of MR shapes or values with increasing temperature. It is noticeable that the MR curve of the G/STO-NIA at $2 \mathrm{~K}$ shows a phenomenon regarded as weak localization (Figure S3d) contrary to that of the G/BFO-NIA. 

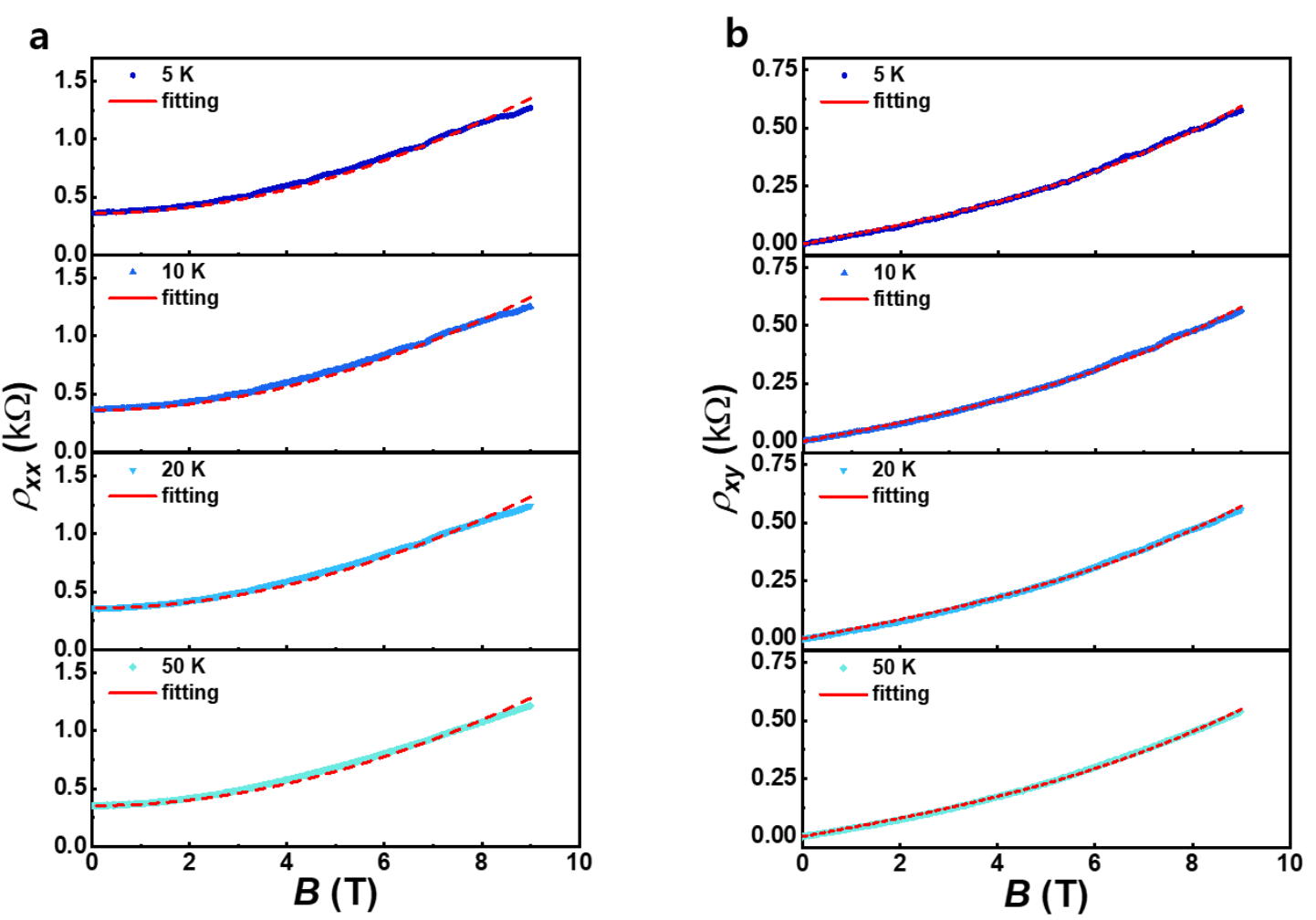

Figure S4. (a) $\rho_{x x}$ and (b) $\rho_{x y}$ of the G/BFO device and their fitting results at temperatures of 5$50 \mathrm{~K}$.

Figures S4a and S4b show the $\rho_{x x}$ and Hall resistivity $\left(\rho_{x y}\right)$ of the G/BFO-NIA, respectively, which are obtained in a magnetic field ranging from $0 \mathrm{~T}$ to $9 \mathrm{~T}$ at the temperatures of $5 \mathrm{~K}, 10 \mathrm{~K}$, $20 \mathrm{~K}$, and $50 \mathrm{~K}$. We performed complete quantitative fits of the experimental data using equations (1) and (2) (mentioned in the manuscript). First, equations (1) and (2) are replaced by the following equations:

$$
\begin{aligned}
& \rho_{x x}(x)=\frac{A+B x^{2}}{e\left(A^{2}+C x^{2}\right)} \\
& \rho_{x y}(x)=\frac{D x+F x^{3}}{e\left(A^{2}+C x^{2}\right)}
\end{aligned}
$$


where $A=n \mu_{\mathrm{e}}+p \mu_{\mathrm{h}}, B=n \mu_{\mathrm{e}} \mu_{\mathrm{h}}{ }^{2}+p \mu_{\mathrm{e}}^{2} \mu_{\mathrm{h}}, C=(p-n)^{2} \mu_{\mathrm{e}}{ }^{2} \mu_{\mathrm{h}}{ }^{2}, D=p \mu_{\mathrm{h}}{ }^{2}-n \mu_{\mathrm{e}}{ }^{2}, F=$ $\mu_{\mathrm{h}}{ }^{2} \mu_{\mathrm{e}}{ }^{2}(p-n)$, and $x=B$. Then, $A$ can be obtained from $\rho_{x x}(0 \mathrm{~T})=\frac{1}{e A}$. Second, by modifying equations (s1) and (s2) to $\frac{\rho_{x y}}{\rho_{x x}}=\frac{D_{0} x+F_{0} x^{3}}{A+B_{0} x^{2}}$ using which the experimental data are fitted, we determine $B_{0}, D_{0}$, and $F_{0}$. We obtain four parameters $\left(n, p, \mu_{\mathrm{e}}\right.$, and $\left.\mu_{\mathrm{h}}\right)$ by solving the set of equations of $A, B_{0}, D_{0}$, and $F_{0}$ simultaneously. Then, $C_{0}$ can be determined using the four parameters. Third, by setting $A$ and $C_{0}$ to their initial values in equations (s1) and (s2), we fit the experimental data $\rho_{x x}(x)$ and $\rho_{x y}(x)$ simultaneously to determine $B_{1}, D_{1}$, and $F_{1}$. Then, we solve the set of equations of $A, B_{1}, D_{1}$, and $F_{1}$ simultaneously to obtain the four parameters $\left(n, p, \mu_{\mathrm{e}}\right.$, and $\left.\mu_{\mathrm{h}}\right)$ and use these parameters to determine $C_{1}$. Fourth, we insert the variables $\left(B_{1}, D_{1}, C_{1}\right.$, and $\left.F_{1}\right)$ obtained through fitting and the constant $A$ in equation (s3) to obtain the error (R) between the experimental data and the fitting results.

$$
(R)^{2}=\sum_{i=1}^{n}\left(\rho_{x x, \text { data }}^{i}-\frac{A+B_{1} x_{i}^{2}}{e\left(A^{2}+C_{1} x_{i}^{2}\right)}\right)^{2}+\sum_{i=1}^{n}\left(\rho_{x y, \text { data }}^{i}-\frac{D_{1} x+F_{1} x_{i}^{3}}{e\left(A^{2}+C_{1} x_{i}^{2}\right)}\right)^{2}
$$

Fifth, we repeat the third and fourth steps until $R$ converges to a minimum value, and finally obtain the optimized parameters $\left(n, p, \mu_{\mathrm{e}}\right.$, and $\left.\mu_{\mathrm{h}}\right)$. The fitting was repeated approximately seven times for each temperature, and the $(R)^{2}$ value at the last step decreased by $\sim 10 \%$ from its initial value. 